\title{
Quantitative trait locus analysis and construction of consensus genetic map for drought tolerance traits based on three recombinant inbred line populations in cultivated groundnut (Arachis hypogaea L.)
}

\author{
B. Gautami $\cdot$ M. K. Pandey $\cdot$ V. Vadez $\cdot$ S. N. Nigam $\cdot$ P. Ratnakumar $\cdot$ \\ L. Krishnamurthy • T. Radhakrishnan • M. V. C. Gowda • M. L. Narasu • \\ D. A. Hoisington · S. J. Knapp • R. K. Varshney
}

Received: 19 August 2011/Accepted: 14 October 2011/Published online: 22 November 2011

(C) The Author(s) 2011. This article is published with open access at Springerlink.com

\begin{abstract}
Groundnut (Arachis hypogaea L.) is an important food and cash crop grown mainly in semi-arid tropics (SAT) regions of the world where drought is the major constraint on productivity. With the aim of understanding the genetic basis and identification of quantitative trait loci (QTL) for drought tolerance, two new recombinant inbred line (RIL) mapping populations, namely ICGS $76 \times$ CSMG 84-1 (RIL-2) and ICGS $44 \times$ ICGS 76 (RIL-3), were used. After screening of 3,215 simple sequence repeat (SSR) markers on the parental genotypes of these populations, two new genetic maps were developed with 119 (RIL-2) and 82 (RIL-3) SSR loci. Together with these maps and the reference map with 191 SSR loci based on TAG
\end{abstract}

Electronic supplementary material The online version of this article (doi:10.1007/s11032-011-9660-0) contains supplementary material, which is available to authorized users.

B. Gautami · M. K. Pandey · V. Vadez ·

S. N. Nigam · P. Ratnakumar · L. Krishnamurthy ·

D. A. Hoisington · R. K. Varshney

International Crops Research Institute for the Semi-Arid

Tropics (ICRISAT), Patancheru 502 324, India

B. Gautami · M. L. Narasu

Institute of Science and Technology, Jawaharlal Nehru

Technological University Hyderabad (JNTUH),

Greater Hyderabad 500 085, India

T. Radhakrishnan

Directorate of Groundnut Research (DGR), Junagadh

362001 , India
$24 \times$ ICGV 86031 (RIL-1), a consensus map was constructed with 293 SSR loci distributed over 20 linkage groups, spanning 2,840.8 cM. As all these three populations segregate for drought-tolerance-related traits, a comprehensive QTL analysis identified 153 main effect QTL (M-QTL) and 25 epistatic QTL (EQTL) for drought-tolerance-related traits. Localization of these QTL on the consensus map provided 16 genomic regions that contained 125 QTL. A few key genomic regions were selected on the basis of the QTL identified in each region, and their expected role in drought adaptation is also discussed. Given that no major QTL for drought adaptation were identified, novel breeding approaches such as marker-assisted recurrent selection (MARS) and genomic selection (GS) approaches are likely to be the preferred approaches for introgression of a larger number of QTL in order to breed drought-tolerant groundnut genotypes.

\author{
M. V. C. Gowda \\ University of Agricultural Sciences, Dharwad \\ 580005 , India \\ S. J. Knapp \\ The University of Georgia, Athens, GA 30602, USA \\ R. K. Varshney $(\bowtie)$ \\ CGIAR-Generation Challenge Programme (GCP), \\ c/o CIMMYT, 06600 Mexico, DF, Mexico \\ e-mail: r.k.varshney@cgiar.org
}


Keywords Peanut - Drought tolerance $\cdot$ Consensus map · QTL analysis · Main-effect QTL · Epistatic QTL - Molecular breeding

\section{Introduction}

Cultivated groundnut or peanut (A. hypogaea L.) is an allotetraploid $(2 \mathrm{n}=4 \mathrm{x}=40)$ crop grown extensively in about 108 countries, with over two-thirds of global production coming from seasonally rainfed areas of tropical, sub-tropical and warm regions of the world. The annual global area of this crop is 23.50 million hectares, with 35.52 million tonnes of production (FAOSTAT 2009). The productivity of groundnut in rainfed areas, where drought is considered to be the major yield-limiting factor (Rucker et al. 1995), is considerably lower than the global average and still lower compared to its productivity in the betterendowed regions. Breeding drought-tolerant cultivars is therefore an important objective in most of the groundnut improvement programs around the world. However, the lack of reliable and rapid tools for screening drought-related traits is the major obstacle in the progress of genetic improvement for drought tolerance in groundnut.

Drought tolerance is a complex trait in which plants have developed the following strategies: escape, avoidance, tolerance and recovery. Most of the efforts in groundnut to date have been directed towards drought escape and drought avoidance (Zhang et al. 2001). The plant either takes advantage of developmental flexibility to match its phenology to the length of the period of soil moisture availability (drought escape), or increases its water absorption ability and decreases its water loss (drought avoidance). Tolerance to drought is likely to be conditioned by many genes under strong environmental influence and thus the networks involved in drought tolerance are quite complex in nature. Selection based on the phenotype would therefore be difficult for such traits (Collins et al. 2008). Since groundnut is grown mostly in semiarid tropics (SAT) environments, which are characterized by short and erratic rainfall and long dry spells, drought avoidance assumes greater importance. Water-use efficiency (WUE) has been considered to be an important drought avoidance trait that concerns using soil water more efficiently for biomass production (Blum 2005; Collins et al. 2008). Raising the
WUE of both irrigated and rainfed crop production is an urgent imperative (Nigam et al. 2005). Transpiration efficiency (TE), an important component of WUE, is also considered to be an important target trait for developing drought-tolerant genotypes for water-limited environments. Surrogate traits for TE, such as specific leaf area (SLA) and SPAD chlorophyll meter reading (SCMR), have been considered in earlier studies (Hubick et al. 1986; Nageswara Rao and Wright 1994; Rebetzke et al. 2002), though concerns have also been raised in some recent studies (Krishnamurthy et al. 2007; Devi et al. 2011). SLA is a measurement of leaf thickness and density, which influence plant water use (Kholová et al. 2010a, b), while SCMR indicates the nitrogen status.

Developing drought-tolerant crop varieties through conventional breeding is time-consuming and laborintensive due to the quantitative nature of drought tolerance and difficulties in selection for drought tolerance (Ribaut et al. 1997). Recent advances in crop genomics offer tools for assisting breeding through identification and introgression of genomic regions associated with drought tolerance to develop improved cultivars with increased drought tolerance using marker-assisted selection (MAS) (Ribaut et al. 1996; Varshney et al. 2005, 2006). To identify the genomic regions suitable for marker-assisted breeding strategies, it is important to establish accurate phenotyping methods followed by development of dense genetic linkage maps/consensus maps and identification of quantitative trait loci (QTL) with traits of interest.

Several studies have reported identification of QTL for drought tolerance or related traits in other crops; however, in the case of groundnut, QTL study for drought tolerance traits has been conducted based on only one mapping population (TAG $24 \times$ ICGV 86031). Comprehensive QTL analysis led to the identification of a total of 117 small main-effect QTL (M-QTL) and 23 epistatic QTL (E-QTL) for drought-related traits (Ravi et al. 2011). In summary, QTL identified based on this mapping population are not suitable for deployment in MAS strategies. When aiming to confirm the involvement of small-effect QTL for drought tolerance, it is essential to undertake similar kinds of drought-tolerance QTL analysis based on some other mapping populations. QTL analysis using other mapping populations may also yield some new QTL which were not identified based on mapping populations studied previously. 
QTL analysis in new mapping populations involves development of new genetic maps. Based on common markers mapped on genetic maps for different mapping populations, it is possible to develop the consensus map (Ablett et al. 2003; Karakousis et al. 2003). Consensus maps provide marker orders for the maximum number of marker loci onto a single map. Keeping in mind the importance of identification of drought-tolerance QTL in different mapping populations and development of a consensus drought-tolerance QTL map, this study used two new recombinant inbred line (RIL) populations, namely ICGS $76 \times$ CSMG $84-1$ and ICGS $44 \times$ ICGS 76. We report here the construction of two new genetic linkage maps, phenotyping and comprehensive QTL analysis (including E-QTL analysis) based on these two mapping populations. In addition, a consensus drought-tolerance QTL map has been developed using genetic and phenotyping data from these two mapping populations with that of the mapping population reported earlier (TAG $24 \times$ ICGV 86031).

\section{Materials and methods}

Mapping populations and DNA isolation

Three recombinant inbred line (RIL) populations, viz. RIL-1 (TAG $24 \times$ ICGV 86031), RIL-2 (ICGS $76 \times$ CSMG 84-1) and RIL-3 (ICGS $44 \times$ ICGS 76), were developed at ICRISAT, Patancheru, India (Electronic Supplementary Material 1). Genetic and QTL maps based on the RIL-1 population have previously been reported (Varshney et al. 2009a; Ravi et al. 2011). RIL-2 and RIL-3, consisting of 177 and 188 RILs, respectively, were used in the present study for developing the genetic maps and QTL analyses.

DNA was extracted from fresh furled leaves of the parental genotypes and from their respective RILs using the modified cetyltrimethylammonium bromide (CTAB) extraction method, as described in Cuc et al. (2008). The DNA quality and quantity were checked on $0.8 \%$ agarose gels and DNA concentration was normalized to $\sim 5 \mathrm{ng} / \mu \mathrm{l}$ for further work.

Trait phenotyping

The RIL-2 population comprising 177 F9 line individuals was phenotyped for transpiration efficiency
(TE, g biomass $\mathrm{kg}^{-1}$ water transpired), transpiration ( $\mathrm{T}, \mathrm{g} \mathrm{plant}^{-1}$ ) and shoot dry weight (ShDW, g plant ${ }^{-1}$ ) during the post-rainy season in 2008 under wellwatered (WW) and water-stress (WS) regimes using the protocols given in Krishnamurthy et al. (2007). In short, plants were grown in $28 \mathrm{~cm}$ diameter pots filled with $10 \mathrm{~kg}$ of Alfisols soil collected from the ICRISAT farm and suitably fertilized. Three seeds for each genotype were planted and seedlings thinned to one healthy seedling per pot at 2 weeks after sowing. T measurements were initiated at 5 weeks after sowing. Pots were saturated with water and left to drain overnight. Plants were then bagged around the stem to avoid soil evaporation. Regular weighing allowed the measurement of plant transpiration. The water-stress treatment was applied by allowing pots to lose no more than $100 \mathrm{~g} \mathrm{day}^{-1}$ (Krishnamurthy et al. 2007). TE was calculated as the ratio of the biomass increase during the experimental period divided by the amount of water used during that time. An extra set of plants was used to assess the biomass before initiating the experiment. In the second season (post-rainy season in 2009), the population was phenotyped for transpiration efficiency (TE, $\mathrm{g}$ biomass $\mathrm{kg}^{-1}$ water transpired), leaf dry weight (LDW, g plant ${ }^{-1}$ ), transpiration $\left(\mathrm{T}, \mathrm{g}\right.$ plant $^{-1}$ ), total dry weight (TDW, $\mathrm{g} \mathrm{plant}^{-1}$, which includes sum of shoot and pod dry weight), SCMR and leaf area $\left(\mathrm{LA}, \mathrm{cm}^{2}\right.$ plant $^{-1}$ ) under well-watered conditions only.

The RIL-3 population was segregated for harvest index (HI) and was then phenotyped in the field under both fully irrigated conditions and intermittent droughtstress conditions. The intermittent drought stress was applied from 40 days after sowing, by skipping the irrigation every other time that the fully irrigated control was watered, so that the amount of water received in the water-stress treatment was about half of that in the fully irrigated control. This RIL-3 population comprising $188 \mathrm{~F} 8$ lines was phenotyped for vegetative weight/plant at harvest $\left(\mathrm{VegWt} / \mathrm{pl}, \mathrm{g}\right.$ plant $\left.^{-1}\right)$, pod weight/plant (PodWt/pl, g plant ${ }^{-1}$ ) and harvest index (HI) during the post-rainy season in 2008 .

Marker polymorphism and analysis

A total of 3,215 simple sequence repeat (SSR) markers available in the public domain and/or accessed through collaborators (ESM 2) were used to screen the parental genotypes of the RIL-2 and RIL-3 
populations. Subsequently, the polymorphic markers identified were used to genotype all the individuals of the respective populations.

PCR reactions and fragment analysis for SSR markers were performed essentially as described in Ravi et al. (2011).

\section{Construction of genetic maps}

Marker segregation data obtained in the RIL-2 and RIL-3 populations were subjected to Chi-squared $\left(\chi^{2}\right)$ testing to examine distortion from the expected 1:1 segregation using Join Map 3.0 (Stam 1993). To assemble linkage groups by maximum-likelihood and to construct the map, Mapmaker/EXP v.3.0 (Lander et al. 1987) was used for the respective mapping populations. Marker clusters were identified using a minimum LOD score of 5.0 for both the mapping populations (RIL-2 and RIL-3) and a maximum recombination fraction $(h)$ of 0.35 . The most likely marker order within each linkage group (LG) was estimated by comparing the log-likelihood of the possible orders of markers using multipoint analysis "Compare" command. The "Try" command was also used to determine the most likely placement of the unlinked markers, and subsequent orders were tested using the "Ripple" command with "Error Detection" and "Use Three Points" options enabled. The distance between neighboring markers were calculated using the multipoint analysis implemented in the "Map" command. The Kosambi mapping function (Kosambi 1944) which incorporates the possibility of crossover interference was used to convert recombination frequencies into map distances in centimorgans $(\mathrm{cM})$.

\section{Quantitative trait locus analysis}

For identification of candidate QTL regions for drought component traits, two types of QTL mapping approaches were used. While the interval mapping (IM) approach was used to identify main-effect QTL (M-QTL), epistatic interaction analysis (EIA) was used to identify epistatic interactions between different QTL regions or epistatic QTL (E-QTL). The most likely locations of QTL and their genetic effects were initially detected by composite interval mapping (CIM; Zeng 1993, 1994) using WinQTL Cartographer, v.2.5 (Wang et al. 2007) as described in Ravi et al. (2011). Similarly, as described in Ravi et al. (2011), the QTLNetwork 2.0 program based on a mixed linear model (Yang et al. 2005) was used to identify epistatic QTL (E-QTL) conditioning drought-related traits. EIA analysis was carried out using Genotype Matrix Mapping (GMM) software v.2.1 (Isobe et al. 2007), www.kajusa.or. jp/GMM) as followed by Ravi et al. (2011).

\section{Construction of consensus map}

The consensus genetic linkage map was constructed by using marker genotyping data for all three individual mapping populations (RIL-1, RIL-2 and RIL-3) using the software MergeMap (Yonghui et al. 2008). In MergeMap, individual maps are first converted to direct acyclic graphs (DAG), which are then merged into a consensus graph on the basis of their shared vertices. MergeMap then attempts to resolve conflicts among the individual maps by deleting a minimum set of marker occurrences. The result of the conflictresolution step is a consensus DAG, which is then simplified and linearized to produce the final consensus map.

\section{Results}

Marker polymorphism and genotyping

A total of 3,221 SSR markers available in the public domain and accessed through collaborators were screened on the parental genotypes of the two new mapping populations, RIL-2 and RIL-3 (ESM 2). Only $126(3.98 \%)$ and $87(2.71 \%)$ markers showed polymorphism between the parental genotypes of RIL-2 and RIL-3, respectively. These polymorphic markers were used for genotyping in the sets of 177 (RIL-2) and 188 (RIL-3) RILs of the respective mapping populations. While genotyping the mapping population RIL-2, segregation data were scored at two loci for two markers (GM2724 and GM2233). As a result, segregation data were obtained for a total of 128 loci for 126 polymorphic markers. For RIL-1, genotyping data were obtained for 215 loci after screening the parental genotypes with 3,215 SSR markers as reported in Ravi et al. (2011).

In summary, after screening a total of 3,215 SSR markers on parental genotypes of three mapping populations, segregation data were obtained for 215 loci on RIL-1, 128 loci on RIL-2 and 87 loci on RIL-3. 
Construction of individual linkage maps

A genetic map comprising 191 SSR loci for RIL-1 was reported in our earlier study (Ravi et al. 2011), and two new genetic maps, based on RIL-2 and RIL-3, have been developed in this study. Genotyping data obtained on RIL-2 and RIL-3, respectively, were checked for segregation ratio using the $\chi^{2}$ test. A total of $58.59 \%$ (RIL-2) and $91.95 \%$ (RIL-3) markers showed the expected 1:1 segregation ratio $(P<$ $0.05)$ and were used to establish the linkage groups (LGs). Using a minimum LOD score of 5.0 and a maximum recombination fraction $(\theta)$ of 0.35 , a total of 119 out of 128 polymorphic SSR loci were integrated onto 20 LGs for the mapping population RIL-2. This genetic map covers a total map distance of 2,208.2 cM and a mean distance of $5.95 \mathrm{cM}$ between markers (ESM 3). The number of markers per LG ranged from two (LG3, LG16 and LG18) to ten (LG7) (ESM 3, ESM 4, ESM 5), while nine markers remained unlinked.

Similarly, using the same LOD score and recombination fraction, a total of 82 out of 87 loci were mapped onto 15 LGs in RIL-3, covering $831.4 \mathrm{cM}$ with an average marker distance of $5.47 \mathrm{cM}$. Five loci remained unlinked and the number of markers per LG ranged from two (LG6, LG8, LG13 and LG14) to 14 (LG7) (ESM 3, ESM 4, ESM 5). Average map distance and average inter-locus distance on the genetic maps developed for all three populations are given in ESM 4. Marker loci on all the maps were given the same name as the primer name used by the source laboratories (ESM 2).

QTL analysis for drought-tolerance traits

Phenotyping of parents and RILs showed moderate variations and low heritability for all the traits studied in both the populations RIL-2 and RIL-3 (ESM 6, ESM 7). It was also observed that all the traits showed continuous distribution, indicating their polygenic nature, except SCMRWW09 (ESM 7) where the phenotypic distribution of RILs was skewed.

Detailed QTL analysis based on genotyping data and phenotyping data on RIL-2 and RIL-3 populations as mentioned above is discussed in the following sections, while results based on the RIL-1 population have been described elsewhere (Ravi et al. 2011).
Identification of main-effect QTL using QTL Cartographer and QTLNetwork

M-QTL analysis based on phenotyping data for the 2 years 2008 and 2009 and genotyping data as mentioned above was conducted using QTL Cartographer and QTLNetwork software.

QTL Cartographer, using the composite interval mapping (CIM) method, detected a total of 24 and three M-QTL in the RIL-2 and RIL-3 populations, respectively. Seven M-QTL for TE, nine M-QTL for T, three M-QTL for TDW and five M-QTL for ShDW were identified with phenotypic variance explained (PVE) of 5.63-18.12\%, 4.83-18.17\%, 6.62-22.39\% and 5.03-22.09\%, respectively. No M-QTL could be detected for SCMR in the RIL-2 population. However, only three M-QTL could be detected for HI measured under well-watered conditions with PVE ranging from 6.39 to $40.10 \%$ in the RIL-3 population. Similarly, using QTLNetwork, a total of seven and two M-QTL were identified in the RIL-2 and RIL-3 populations, respectively. In the RIL-2 population, three M-QTL for TE with PVE ranging from 3.31 to $4.25 \%$ were detected, along with a single M-QTL each for $\mathrm{T}$ (3.21\% PVE), TDW (6.04\% PVE), ShDW (5.50\% PVE) and SCMR (2.51\% PVE). However, in the RIL3 population, only a single M-QTL each for $\mathrm{HI}(3.29 \%$ PVE) and VegWt/pl (2.28\% PVE) could be detected (Table 1, ESM 8, ESM 9).

Identification of epistatic QTL by QTLNetwork and genotype matrix mapping

Drought tolerance is a polygenic trait which involves multiple gene interactions. Therefore, the present study also aimed to identify epistatic QTL (E-QTL) which arise due to interactions among the M-QTL detected for different drought-related traits using QTLNetwork and genotype matrix mapping (GMM) software. Using the QTLNetwork program, a total of ten E-QTL were detected in two mapping populations (RIL-2 and RIL-3). Of these, two E-QTL each were detected for TE (PVE 2.44-2.91\%) and T (PVE 7.29-9.01\%) and one E-QTL each for ShDW (PVE $7.64 \%)$, LA (11.09\%), LDW (7.65\%), TDW (8.89\%), SCMR (4.77\%) and VegWt/pl (7.66\%) (ESM 10). Two examples of marker loci interactions for transpiration efficiency under stress in RIL-2 and pod weight 
Table 1 Main effect QTL (M-QTL) for drought tolerance identified by QTL cartographer and QTLNetwork

\begin{tabular}{|c|c|c|c|c|}
\hline \multirow[t]{2}{*}{ Traits } & \multicolumn{2}{|c|}{ QTL cartographer } & \multicolumn{2}{|l|}{ QTLNetwork } \\
\hline & $\begin{array}{l}\text { No. of QTL } \\
\text { identified }\end{array}$ & $\begin{array}{l}\text { PVE } \\
\left(R^{2} \%\right)\end{array}$ & $\begin{array}{l}\text { No. of QTL } \\
\text { identified }\end{array}$ & $\begin{array}{l}\mathrm{PVE} \\
\left(R^{2} \%\right)\end{array}$ \\
\hline \multicolumn{5}{|l|}{$R I L-2$} \\
\hline Transpiration efficiency (TE) & 7 & $5.63-18.12$ & 3 & $3.31-4.75$ \\
\hline Transpiration $(\mathrm{T})$ & 9 & $4.83-18.17$ & 1 & 3.21 \\
\hline Total dry weight (TDW) & 3 & $6.62-22.39$ & 1 & 6.04 \\
\hline Shoot dry weight (ShDW) & 5 & $5.03-22.09$ & 1 & 5.5 \\
\hline $\begin{array}{l}\text { SPAD chlorophyll meter } \\
\text { readings (SCMR) }\end{array}$ & - & - & 1 & 2.51 \\
\hline \multicolumn{5}{|l|}{$R I L-3$} \\
\hline Harvest index (HI) & 3 & $6.39-40.10$ & 1 & 3.29 \\
\hline Vegetative weight/plant (VegWt/pl) & - & - & 1 & 2.28 \\
\hline
\end{tabular}

under well-watered conditions in RIL-3 are shown in Fig. 1 and ESM 11, respectively.

Interaction of QTL for two and three loci using the GMM program detected a total of 37 E-QTL in RIL-2 and 26 E-QTL in RIL-3 populations. A total of 18 E-QTL for TE (PVE 12.67-44.77\%), three for T (PVE 15.8-56.56\%), six for ShDW (PVE 12.69-18.72\%), two each for LDW (PVE 29.99-30.87\%) and TDW (PVE 34.07-35.32\%), three for SCMR (PVE 36.33-44.69\%), four for VegWt/pl (PVE 9.94-13.28\%), ten for PodWt/ pl (PVE 23.69-36.02\%) and 12 for HI (PVE $8.42-15.11 \%$ ) were identified. All the E-QTL obtained above involved three loci interactions (ESM 12), while only one E-QTL obtained for ShDW with PVE $14.59 \%$ involved two loci interactions (Table 2). Interestingly, the number of E-QTL identified and PVE observed by QTLNetwork were found to be very low compared to GMM.

\section{Construction of consensus map}

Genetic maps developed for the three populations (RIL-1, RIL-2 and RIL-3) segregating for droughttolerance traits were used for developing a consensus map. It is important to mention that all three maps were constructed with Mapmaker/EXP v.3.0 (Lander et al. 1987) using the same mapping functions. Fortynine loci were common to the genetic maps based on RIL-1 and RIL-2 populations, 33 to the genetic maps based on RIL-1 and RIL-3 populations, 40 to the genetic maps based on RIL-2 and RIL-3 populations, while 13 markers were common to all three maps. Using these common markers, MergeMap was used to develop the consensus map. In this context, the densest genetic map based on the RIL-1 population, with the maximum number of mapped loci (191), was taken as a framework map for combining mapped marker loci from the other two maps based on the RIL-2 and RIL-3 populations.

The integration of different LGs from individual maps to develop the consensus map is given in ESM 13. Based on the common markers and the comparison between individual maps, it was observed that most of the LGs were consistent among the individual maps, with few exceptions (ESM 14). Details on the comparison of different LGs of the consensus map with the three different maps in terms of mapped loci, map length, map density and inter-locus gap distance are given in ESM 3.

In brief, the consensus map developed in this study consists of a total of 293 SSR marker loci with a total distance of 2,840.8 cM on 20 LGs (Fig. 2). The LGs in the consensus map ranged from 6.3 (LG_AhXX) to 293.37 cM (LG_AhIV) with a mean of $142.04 \mathrm{cM}$. The number of markers per LG ranged from two (LG_AhXX) to 31 (LG_AhVII) (ESM 4). Out of 293 mapped loci, 65.19\% (191 loci) of marker intervals were smaller than $10 \mathrm{cM}, 26.90 \%$ (79 loci) were between 10 and $30 \mathrm{cM}$, and $7.85 \%$ (23 loci) were greater than $30 \mathrm{cM}$ (ESM 13).

Mapping M-QTL and E-QTL onto the consensus map

In addition to the $36 \mathrm{M}$-QTL identified in RIL-2 and RIL-3, a total of 117 M-QTL detected in RIL-1 


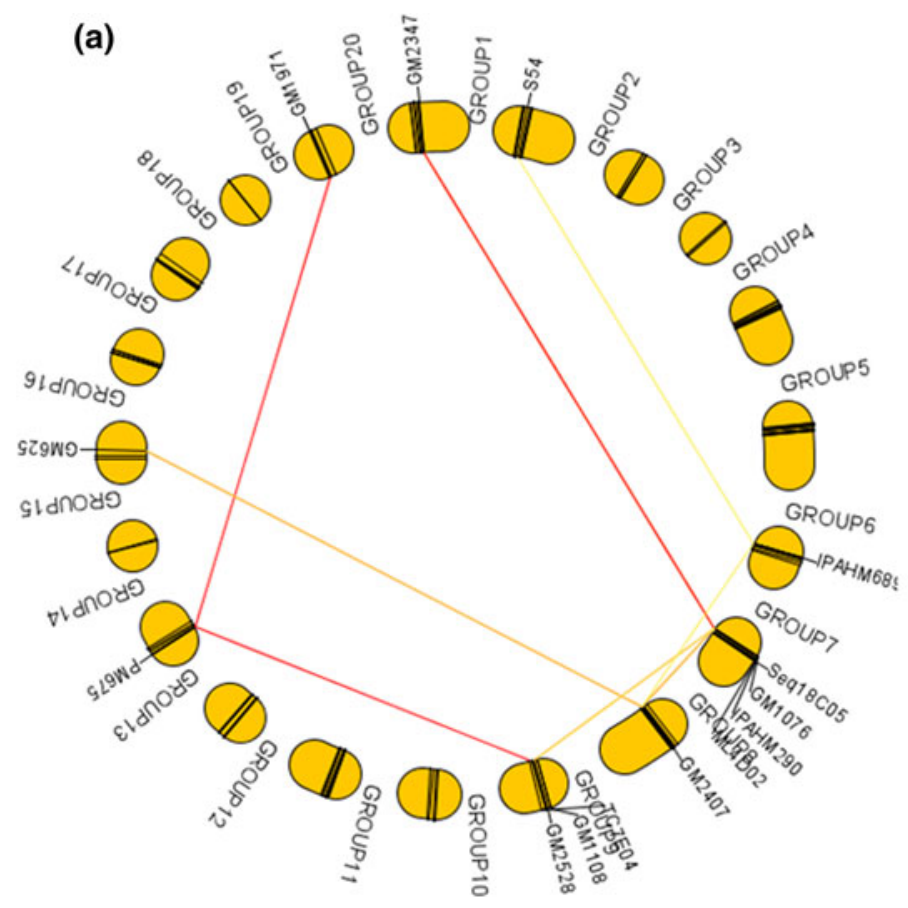

(b)

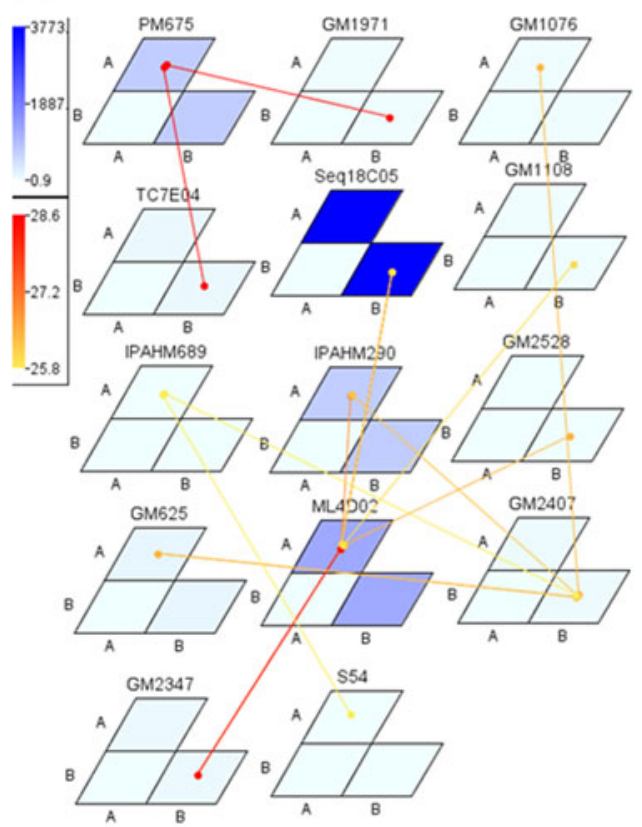

Fig. 1 An example of marker-loci interaction for transpiration efficiency (TEWS) in the RIL-2 population detected by Genotype Mapping Matrix (GMM) software. a Graphical presentation of three-locus interactions and their positions on the genetic linkage map. Linkage groups are arranged in tandem as a circle and triangles in the circle represent the interaction of

(Varshney et al. 2009a; Ravi et al. 2011) were taken into consideration to place them onto the newly developed consensus map. Altogether, a total of 153 M-QTL identified from the three mapping populations for the drought component traits were placed onto 16 of the 20 LGs, while no M-QTL could be mapped on four LGs (LG_AhII, LG_AhXV, LG_AhXVIII and $\left.L G \_A h X X\right)$ on the newly constructed consensus map. Ten or more M-QTL were identified on LG_AhV (21), LG_AhVII (19), LG_AhXI (16), LG_AhX (14), LG_AhIV (12), LG_AhVIII (10), LG_AhXIII (10) and LG_AhXVII (10). Less than ten M-QTL were detected on LG_AhIX (8), LG_AhIII (6), LG_AhXIX (5), LG_AhVI (4), LG_AhXII (4), LG_AhI (3) and LG_AhXVI (3), and a single M-QTL on LG_AhXIV (Fig. 2).

A total of 25 E-QTL identified for the drought component traits from the three mapping populations were distributed on 15 LGs of the newly developed consensus map. No E-QTL could be detected on five linkage groups, viz.LG_AhVIII, LG_AhX, LG_AhXV, a three-locus combination. b Graphical presentation of interacting loci and allele type by genotype matrices (GMs) and a genotype matrix network (GMN). Significant locus/allele combinations of three interacting loci are shown by GMs and GMN. Matrices and connecting lines indicate GMs and GMNs, respectively. (Details are available in ESM 12.)

LG_AhXVII and LG_AhXX. Five E-QTL were detected in LG_AhIII, four in LG_AhVII and three each in LG_AhIX, LG_AhXI, LG_AhXIII and LG_AhXVI. Two E-QTL each were detected in LG_AhII, LG_AhIV, LG_AhV and LG_AhVI, and a single E-QTL each in LG_AhI, LG_AhXII, LG_AhXIV, LG_AhXVIII and LG_AhXIX.

A total of 178 QTL (153 M-QTL and 25 E-QTL) associated with 25 traits based on the three mapping populations (RIL-1, RIL-2 and RIL-3) were placed onto the newly developed consensus map. Several QTL clusters were found scattered on 14 LGs (LG_AhIII, LG_AhIV, LG_AhV, LG_AhVI, LG_AhVII, LG_AhVIII, LG_AhIX, LG_AhX, LG_AhXI, LG_AhXII, LG_AhXIII, LG_AhXVI, LG_AhXVII and LG_Ah$\mathrm{XIX)}$ of the consensus map (Table 3 ). The region GM1949-TC7E04 (29.3 cM) on LG_AhIII harboured five QTL for LDW, T, ShDW, TDW and TE traits. The TC1D02-TC3E05 (31 cM) region and pPGSeq19D06PM418 (37.8 cM) region on LG_AhIV harboured seven and six QTL, respectively, for HaulmWt, SCMR, TDW, 
VegWt/pl, SLA, ShDW, canopy conductance (ISC) and T. LG_AhV had two clusters, viz. GM630-TC6E01 (39.2 cM) with 18 QTL for PodWt, SeedWt, TDM, HaulmWt, TE, T and ISC, and GM2584pPGSSeq17F06 (74 cM) with five QTL for HI, T and TDW. PM375-GM1867 (25.1 cM) on LG_AhVII harboured 16 QTL for LA, SeedWt, PodWt, TDM, T, SLAHar, Biomass, ShDW, DWInc and TE. On LG_Ah VIII, nine QTL for the traits SLA, Haulmwt, SCMR, ShDW and TE are harboured in the region pPGPSeq3A06-IPAHM406 (50.4 cM). Similarly, five QTL were present in the pPGPSeq2B09-GM634 region $(17.9 \mathrm{cM})$ on LG_AhIX for SCMR, ISC and LA traits. LG_AhXI harboured two clusters, viz. genomic region GM2350-GM2724a (52.2 cM) with four QTL for the traits initial DW, SLA and Delta13C, while the GM1971b-TC4H02 region (48.9 cM) harboured twelve QTL for T, HaulmWt, ISC, Biomass, SLA, SCMR, TE and TDM. Nine QTL were found on LG_AhXIII in the GM1911-PM733b region $(28.3 \mathrm{cM})$ for the traits SLA, SCMR, T and ShDW. Six QTL were clustered on LG_AhXVI in the GM2050-GM1494 region $(39.0 \mathrm{cM})$ for $\mathrm{HI}, \mathrm{VegWt} / \mathrm{pl}$, TDW, PodWt/pl and ShDW, while nine QTL were mapped on LG_AhXVII in the region GM1418-S11 (34.3 cM) for the traits HI, SLA and SCMR. Similarly, genomic region GM1021-GM1570 (21.3 cM) harboured three QTL on LG_AhXIX for TDW, SCMR and T.
Fig. 2 A consensus genetic map with M-QTL and E-QTL for drought component traits in cultivated groundnut. M-QTL and E-QTL for different traits are shown on the right-hand side of linkage groups with blue, green and red bars indicating mapping of these QTL in RIL-1, RIL-2 and RIL-3, respectively

\section{Discussion}

Marker polymorphism and genetic maps

Screening of a large number of SSR markers $(3,215)$ on the parental genotypes of all three mapping populations showed a very low level of polymorphism (RIL-1: 6.69\%, RIL-2: 3.91\% and RIL-3: 2.7\%). This may be attributed mainly to two reasons: (1) narrow genetic diversity in the cultivated groundnut gene pool (Young et al. 1996; Varshney et al. 2009a; Hong et al. 2010; Ravi et al. 2011, Sarvamangala et al. 2011), and (2) highly conserved region (cDNA) as the source of the majority ( $94 \%$ expressed sequence tag-derived) of the SSR markers used (Varshney et al. 2005). Eight SSR markers (TC3G01, pPGSSeq9H08, IPAHM108, PM733, GM1971, GM1992, GM723 and GM635) in the RIL-1 population (Varshney et al. 2009a; Ravi et al. 2011) and two markers (GM2724 and GM2233) in the RIL-2 population amplified more than one polymorphic loci. Amplification of more than one locus, due to the polyploid nature of the crop, has been
Table 2 Summary of epistatic interactions at three and two loci identified with genotype matrix mapping (GMM)

PVE phenotypic variance explained

\begin{tabular}{|c|c|c|c|c|}
\hline \multirow[t]{2}{*}{ Traits } & \multicolumn{2}{|l|}{$\begin{array}{l}\text { Three-locus } \\
\text { interactions }\end{array}$} & \multicolumn{2}{|c|}{ Two-locus interactions } \\
\hline & $\begin{array}{l}\text { No. of QTL } \\
\text { indentified }\end{array}$ & $\operatorname{PVE}\left(R^{2} \%\right)$ & $\begin{array}{l}\text { No. of QTL } \\
\text { indentified }\end{array}$ & $\operatorname{PVE}\left(R^{2} \%\right)$ \\
\hline \multicolumn{5}{|l|}{$R I L-2$} \\
\hline Transpiration efficiency (TE) & 18 & $12.67-44.77$ & - & - \\
\hline Transpiration $(\mathrm{T})$ & 3 & $15.8-56.56$ & - & - \\
\hline Shoot dry weight (ShDW) & 6 & $12.69-18.72$ & 1 & 14.59 \\
\hline Leaf area (LA) & 2 & $29.99-30.87$ & - & - \\
\hline Leaf dry weight (LDW) & 2 & $29.99-30.87$ & - & - \\
\hline Total dry weight (TDW) & 2 & $34.07-35.32$ & - & - \\
\hline $\begin{array}{l}\text { SPAD chlorophyll meter } \\
\text { readings (SCMR) }\end{array}$ & 3 & $36.33-44.69$ & - & - \\
\hline \multicolumn{5}{|l|}{$R I L-3$} \\
\hline Vegetative weight/plant (VegWt/pl) & 4 & $9.94-13.28$ & - & - \\
\hline Pod weight/plant (PodWt/pl) & 10 & $23.69-36.02$ & - & - \\
\hline Harvest index (HI) & 12 & $8.42-15.11$ & - & - \\
\hline
\end{tabular}



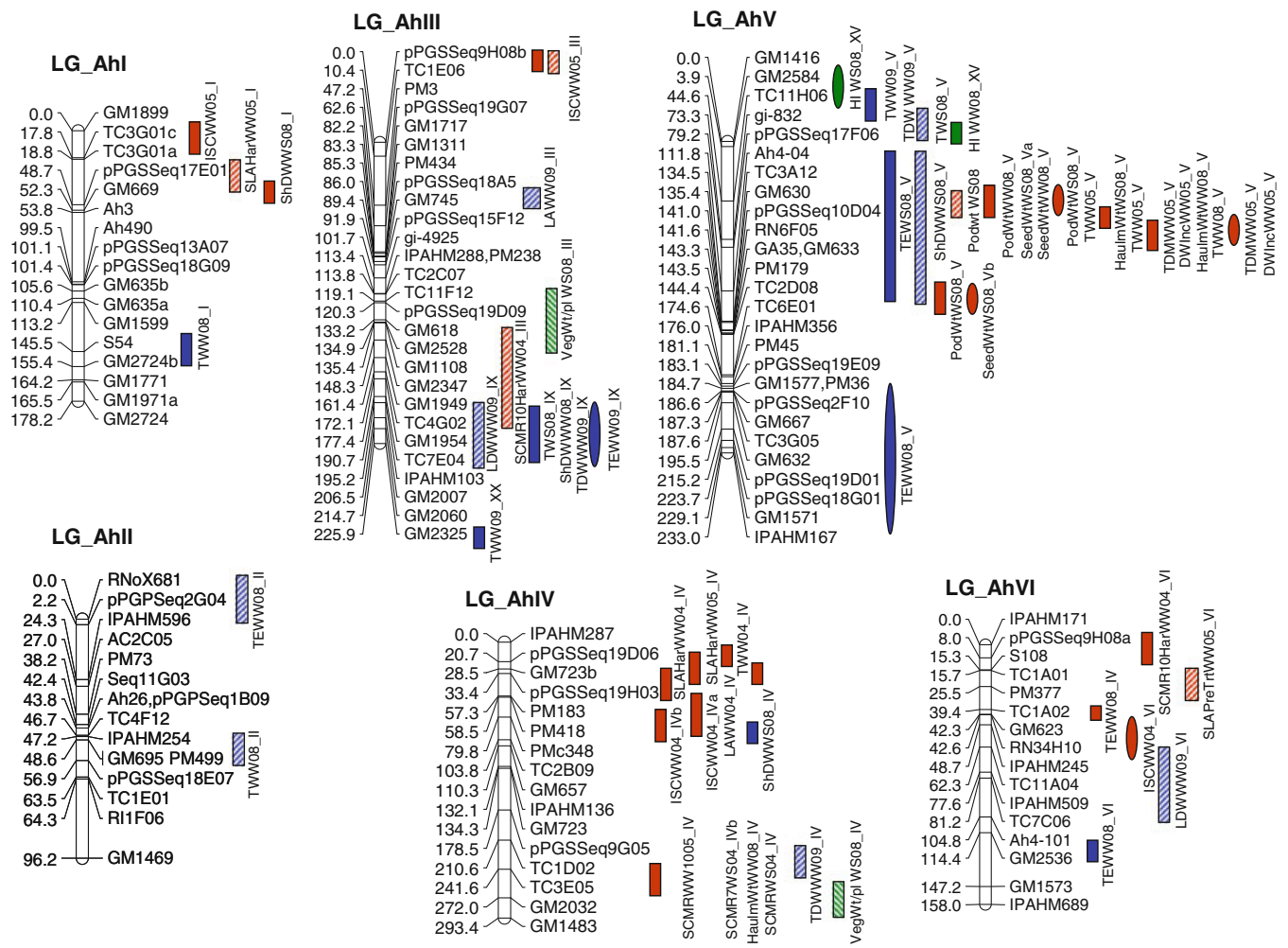

\section{LG_AhVII}
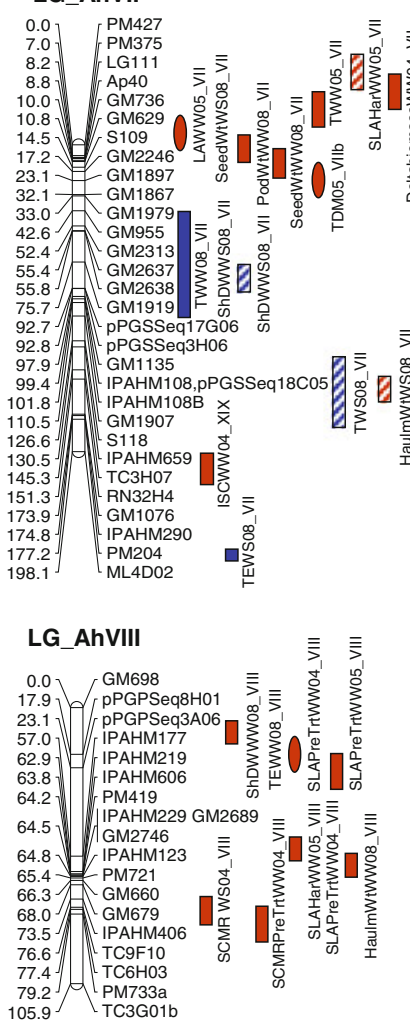

\section{LG_AhIX}

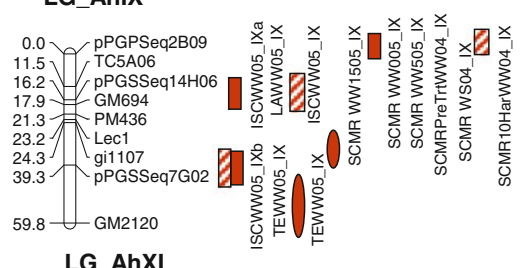

LG_AhXI

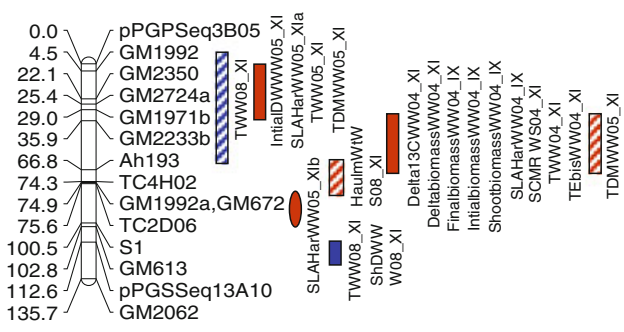

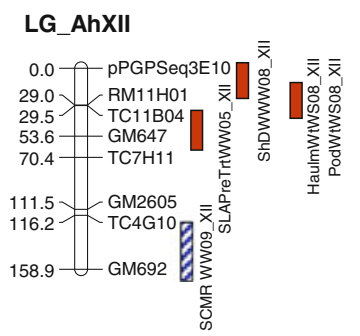




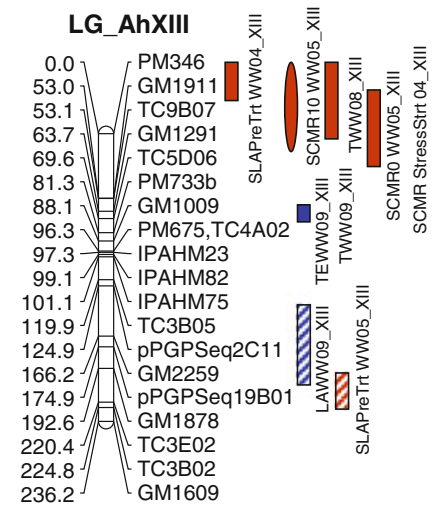

\section{LG_AhXIV}

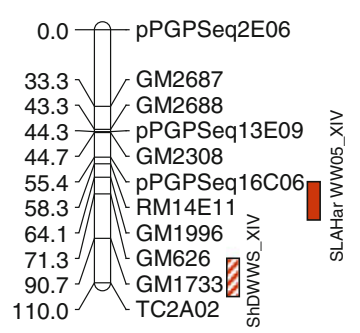

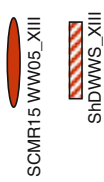

LG_AhXVI

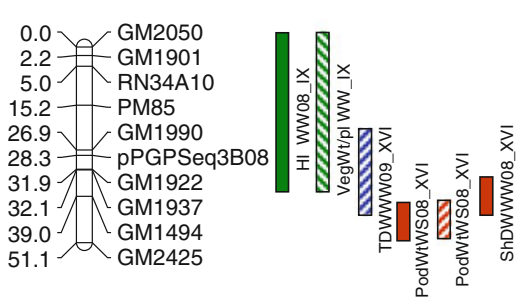

\section{LG_AhXVIII}
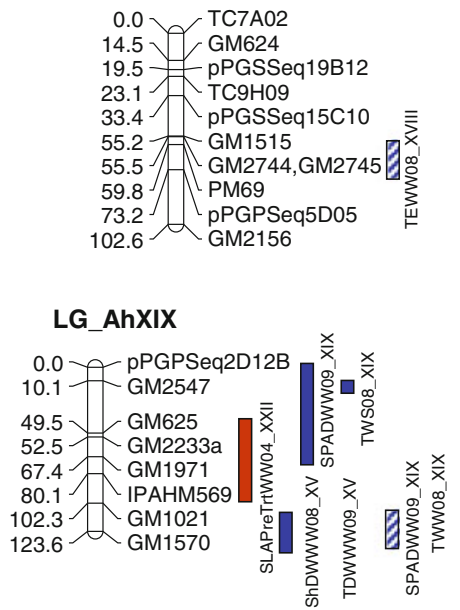

LG_AhXX
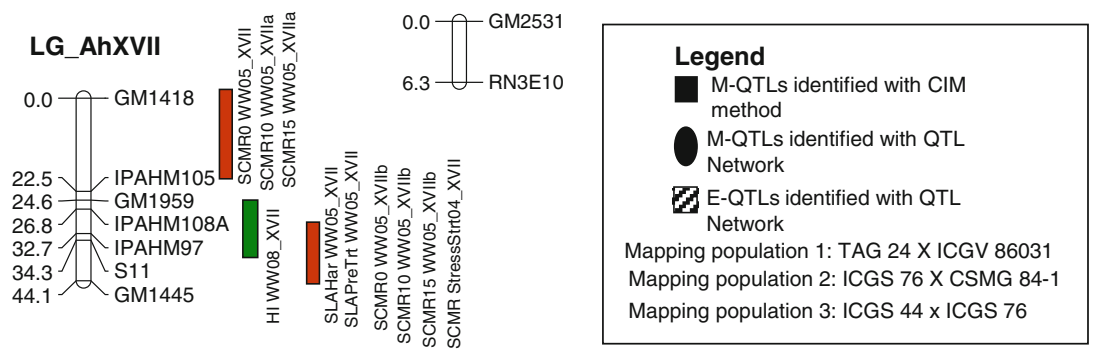

Fig. 2 continued

reported in earlier studies (Hopkins et al. 1999; Krishna et al. 2004; Kottapalli et al. 2007; Varshney et al. 2009a, b; Ravi et al. 2011, Hong et al. 2010). This also suggests variability between genomes for these loci and their potential use in comparative mapping between the AA and BB genomes.

Recently, some genetic maps have been developed in cultivated groundnut (Varshney et al. 2009a; Hong et al. 2010; Khedikar et al. 2010; Ravi et al. 2011; Sarvamangala et al. 2011), but only one population, namely TAG $24 \times$ ICGV 86031, has been used for developing the genetic map and QTL analysis for drought-tolerance traits. In this study, two new RIL populations, namely ICGS $76 \times$ CSMG $84-1$ and ICGS $44 \times$ ICGS 76, segregating for drought tolerance were used to develop two new genetic maps. Together with the genetic map for RIL-1 (Ravi et al. 2011), three genetic maps have now become available for mapping populations segregating for droughttolerance traits.
M-QTL and E-QTL for drought component traits

Drought tolerance is one of the major constraints on productivity in groundnut and a critical understanding of component traits enhancing adaptability towards drought is vital for improving cultivars, since selection based on phenotypic data is not sufficient and reliable due to very strong environmental influence on the trait. To overcome this problem, genomic regions associated with drought-tolerance-related traits can be utilized to develop drought-tolerant varieties through molecular breeding approaches. In this context, QTL analysis based on the RIL-1 population for droughttolerance-related traits showed involvement of several M-QTL and a large number of E-QTL for drought tolerance (Varshney et al. 2009a; Ravi et al. 2011). To validate the results obtained in these previous studies or to identify new QTL, if possible, QTL analysis for drought-tolerance-related traits was undertaken on the RIL-2 and RIL-3 populations in the present study. 
Table 3 QTL clusters identified for biomass, SCMR, yield and drought-related traits

\begin{tabular}{|c|c|c|c|c|c|c|}
\hline $\begin{array}{l}\text { Cluster } \\
\text { no. }\end{array}$ & LGs & Marker interval & $\begin{array}{l}\text { Position } \\
\text { (cM) }\end{array}$ & $\begin{array}{l}\text { No. of } \\
\text { QTL }\end{array}$ & Traits & $\begin{array}{l}\mathrm{PVE} \\
\left(R^{2} \%\right)\end{array}$ \\
\hline 1 & LG_AhIII & GM1949-TC7E04 & 29.3 & 5 & LDW, T, ShDW, TDW, TE & $3.64-22.39$ \\
\hline 2 & LG_AhIV & pPGSSeq19D06-PM418 & 37.8 & 6 & SLA, ISC04, T, ShDW & $3.91-22.24$ \\
\hline 3 & LG_AhIV & TC1D02-TC3E05 & 31.0 & 7 & $\begin{array}{l}\text { HaulmWt, SCMR, TDW, } \\
\text { VegWt/pl }\end{array}$ & $5.06-33.36$ \\
\hline 4 & LG_AhV & $\begin{array}{l}\text { GM2584- } \\
\text { pPGSSeq17F06 }\end{array}$ & 74.0 & 5 & HI, T, TDW & $6.91-7.29$ \\
\hline 5 & LG_AhV & GM630-TC6E01 & 39.2 & 18 & $\begin{array}{l}\text { T, TE, ShDW, PodWt/pl, } \\
\text { SeedWt, HaulmWt, TDM, } \\
\text { DWInc, }\end{array}$ & $1.7-13.44$ \\
\hline 6 & LG_AhVII & PM375-GM1867 & 25.1 & 16 & $\begin{array}{l}\text { LA, SeedWt, PodWt/pl, TDM, } \\
\text { T, SLAHar, Biomass, ShDW, } \\
\text { DWInc, TE }\end{array}$ & $2.93-9.85$ \\
\hline 7 & LG_AhVIII & $\begin{array}{l}\text { pPGPSeq3A06- } \\
\text { IPAHM406 }\end{array}$ & 50.4 & 9 & $\begin{array}{l}\text { SLA, HaulmWt, SCMR, ShDW, } \\
\text { TE }\end{array}$ & $3.90-9.87$ \\
\hline 8 & LG_AhIX & pPGPSeq2B09-GM634 & 17.9 & 5 & SCMR, ISC, LA & $6.23-10.49$ \\
\hline 9 & LG_AhX & GM2444-IPAHM165 & 25.5 & 4 & SCMR & $7.10-12.15$ \\
\hline 10 & LG_AhX & TC9F04-TC4D09 & 16.5 & 7 & $\begin{array}{l}\text { SCMR, PodWt/pl, HaulmWt, } \\
\text { LA, TE }\end{array}$ & $4.67-7.74$ \\
\hline 11 & LG_AhXI & GM2350-GM2724a & 52.2 & 4 & InitialDW, SLA, Delta13C04 & $4.19-20.32$ \\
\hline 12 & LG_AhXI & GM1971b-TC4H02 & 48.9 & 12 & $\begin{array}{l}\text { T, HaulmWt, ISC, Biomass, SLA, } \\
\text { SCMR, TE, TDM }\end{array}$ & $3.44-12.60$ \\
\hline 13 & LG_AhXIII & GM1911-PM733b & 28.3 & 9 & SLA, SCMR, T, ShDW & $3.11-13.96$ \\
\hline 14 & LG_AhXVI & GM2050-GM1494 & 39.0 & 6 & $\begin{array}{l}\text { HI, VegWt/pl, TDW, PodWt/pl, } \\
\text { ShDW }\end{array}$ & $6.62-40.10$ \\
\hline 15 & LG_AhXVII & GM1418-S11 & 34.3 & 9 & SCMR, HI, SLA & $5.41-19.53$ \\
\hline 16 & LG_AhXIX & GM1021-GM1570 & 21.3 & 3 & TDW, SCMR, T & $2.51-9.87$ \\
\hline
\end{tabular}

$P V E$ phenotypic variance explained

In order to undertake comprehensive analysis, two programs, namely QTL Cartographer and QTLNetwork, were used for identification of M-QTL. Similarly, QTLNetwork and GMM programs were utilized to study environmental interactions between different loci (E-QTL). Using these programs, a total of $36 \mathrm{M}$-QTL and $10 \mathrm{E}-\mathrm{QTL}$ were identified for drought-related traits in the RIL-2 and RIL-3 mapping populations. It is important to mention here that the M-QTL identified by QTL Cartographer were also identified by QTLNetwork. Also, the numbers of QTL identified by QTLNetwork were comparatively less than those identified by QTL Cartographer. Similar results were also observed in earlier studies in RIL-1 (Ravi et al. 2011). The M-QTL identified for TE on LG_Ah VI, T on LG_Ah IX, TDW on LG_Ah $\mathrm{V}$ and ShDW on LG_Ah IX for RIL-2, were identified by both the programs (ESM 6). QTL identified by both the programs may be considered to be more reliable than those which are detected by only one program. The value of such QTL, however, can be confirmed only by validating/assessing them in multilocation trials, different genetic backgrounds, etc. Taking into account the results of all three RIL populations, the majority of M-QTLs had moderate additive effects. The combination of these favorable loci derived from both the tolerant (positive additive effect) and the susceptible (negative effect) parents may confer more tolerance to drought. Alleles that improve the trait which are derived from agronomically inferior parents have also been identified for several plant species (Xiao et al. 1998; Frary et al. 2004; Yoon et al. 2006).

The majority of the studies suggested that quantitative variation is determined by a few QTL with a relatively large effect and a large number of QTL with 
smaller effects. Apart from M-QTL, E-QTL which arise due to interactions of different loci in a particular cross also play a significant role in controlling a particular trait (Jannink 2007; Isobe et al. 2007). Therefore, to detect such interactions and QTL coordinations in the present study, epistatic interaction analysis (EIA) undertaken with GMM and QTLNetwork revealed several E-QTL in the two populations (RIL-2 and RIL-3). GMM detected a total of 63 interactions among three loci and only one interaction between two loci for different drought component traits. As expected, the number of E-QTL identified by GMM was greater than the M-QTL. Furthermore, the PVE of these QTL interactions was comparatively higher than the M-QTL. Similar results were also observed in the earlier studies for RIL-1 in groundnut (Ravi et al. 2011) and for plant persistency in rye (Klimenko et al. 2010). This clearly indicates the importance of these interactions for a complex trait such as drought tolerance which is strongly influenced by the environment. Hence, in addition to considering M-QTL (which are fewer in number), selection of the interacting loci (E-QTL) when improving drought tolerance is vital.

Taking into consideration the results of all three RIL populations, it is evident that drought tolerance in groundnut is governed by a large number of M-QTL and E-QTL, each with a small phenotypic variation. Stacking of all these minor QTL is not possible through marker-assisted backcrossing (MABC) for the improvement of drought tolerance in groundnut, as MABC can only be successful in transferring a few major QTL from one genetic background to another (Ribaut et al. 2010). Therefore, alternative and more efficient approaches which allow selection for several QTL with small effects (Ribaut and Ragot 2007; Bernardo 2008; Varshney and Dubey 2009) such as marker-assisted recurrent selection (MARS) or genomic selection (GS) will be more useful for the improvement of drought tolerance in groundnut.

\section{Consensus map for cultivated groundnut}

Development of a consensus map is very useful in such crops like groundnut where a high-density genetic map is not available. To achieve this, two individual maps developed from RIL-2 and RIL-3 in the current study, along with the map developed from the RIL-1 population (Ravi et al. 2011), were used for development of consensus map. The newly developed consensus map consists of 293 SSR loci distributed over 20 LGs. Fourteen out of 20 LGs possessed more than ten markers. The observed total map distance of the newly developed consensus map $(2,840.8 \mathrm{cM})$ was almost equal to the expected genome length of the groundnut genome $(2,800 \mathrm{Mb} / 1 \mathrm{C})$ representing the random distribution of SSR markers across the whole genome. The markers placed on the consensus map were consistent with respect to order of the LGs with the map developed earlier by Ravi et al. (2011), with a few minor differences. This conservative property of the cultivated genome makes the consensus map reliable and successful. This consensus map has removed large gaps present in the individual maps, except in LGs where the poor coverage is due to lack of polymorphic markers in those regions.

To the best of our knowledge, this newly developed consensus map with an average density of $9.96 \mathrm{cM}$ per marker is the first SSR-rich dense consensus map for cultivated groundnut. A similar attempt was made by Hong et al. (2010) and they developed a composite map for tetraploid groundnut with 175 loci using three mapping populations with a total map distance of $885.4 \mathrm{cM}$. For comparable areas, the size of the consensus map developed in the present study was consistently larger than the composite map developed by Hong et al. (2010), which may be due to the use of different programs for development of the consensus map. Moreover, this consensus map was more dense and accurate because all the maps were developed at the same centre (ICRISAT, India) and by using the same set of SSR markers $(3,221)$ for studying marker polymorphism among the parental genotypes. Furthermore, the present consensus map also has the merit of being the first SSR-based consensus map for drought-related traits, as all three populations were segregating for drought-related traits which allowed us to place all the mapped QTL onto the consensus map.

The present consensus map possesses a large number of markers spanning the full genome that can be used to genotype individuals for detecting recombinants, fixing loci, restoring a recurrent genetic background, assembling complex genotypes in complex crosses (Gupta et al. 1999; Somers et al. 2004), comparative mapping and map-based cloning. Future prospects include adding more SSR, single nucleotide polymorphism and Diversity Arrays Technology markers to the consensus map, thus producing a highly 
saturated map and one which helps in a thorough alignment to the physical map of groundnut as well as implementation of the map in several molecular breeding activities in groundnut.

\section{Candidate genomic regions for drought tolerance}

Since all three mapping populations were segregating for drought-related traits, it was worthwhile placing all the QTL identified in the individual maps onto the newly developed consensus map. A total of 178 QTL (153 M-QTL and 25 E-QTL) associated with 25 drought- and yield-related traits were found distributed on 14 LGs. Interestingly, several of these QTL were found clustered in 16 specific genomic regions.

The genomic region $(23.9 \mathrm{cM})$ bracketed by PM375 and GM1867 markers on LG_AhVII possessed 16 QTL for traits LA, SeedWt, PodWt/pl, TDM, T, SLAHar, Biomass, ShDW, DWInc, ShDW and TE. Likewise the GM630-TC6E01 (39.2 cM) genomic region on LG_Ah V contained 18 QTL for traits such as T, TE, ShDW, PodWt/pl, SeedWt, HaulmWt, TDM and DWInc. These regions have QTL for yield and yield component from the field experiment under mild stress with co-mapping of seed weight QTL under WW and WS conditions, and also co-mapping of growth attributes from other phenotyping experiments. Co-mapping of TE QTL $(39.2 \mathrm{cM})$ from an earlier study in the GM630TC6E01 region reconfirms the hypothesis that TE would contribute under situations of mild water stress (Ratnakumar and Vadez 2011). The GM1971bTC4H02 region on LG_AhXI $(48.9 \mathrm{cM})$ harbored 12 QTL for T, HaulmWt, carbon discrimination ratio, biomass, SLA, SCMR, TE and TDM, and is interpreted as being a "growth" region. Interestingly, three out of these four biomass clusters also harbored yield and yield component QTL, which is explained by the mild stress in that field experiment. Our interpretation is that under such conditions genotypes favoring plant growth are likely to achieve higher yields. Similarly, four clusters harbored a total of 26 QTL for SCMR on LG_AhIX (17.9 cM, pPGPSeq2B09-GM634), LG_AhX (25.5 cM, GM2444-IPAHM165), LG_AhXIII (28.3 cM, GM1911-PM733b) and LG_AhXVII (34.3 cM, GM1418-S11). Two clusters also harbored QTL related to leaf characteristics, including leaf area and leaf thickness but also leaf conductance and plant transpiration on LG_AhIV and LG_AhVII. These clusters are particularly important since QTL for leaf conductance and transpiration condition, the rate at which the plant would use a limited water resource, are present in these regions. The pPGPSeq2B09-GM634 region harboured QTL for SCMR, canopy conductance (ISC) and LA. We predict this region to control leaf nitrogen status in conjunction with the leaf expansion processes (more leaf expansion leading to less $\mathrm{N} \mathrm{cm}^{-2}$ and consequently a lower SCMR reading). Both of these traits are indirectly involved in setting the level of canopy conductance that seems to play an important role in specific drought conditions. The region on LG_AhXIII in GM1911-PM733b with six QTL for the traits SLA, SCMR, T and ShDW is interpreted as another region controlling the nitrogen status of the plant.

The region on LG_AhXVI at GM2050-GM1494 (39 $\mathrm{cM}$ ) with six clustered QTL for HI, VegWt/pl, TDW, PodWt/pl and ShDW traits is particularly interesting because it harbours HI QTL from RIL-3, dry weight (TDW and ShDW) QTL from RIL-2 and yield and shoot QTL from RIL-1. As mentioned above, recent findings indicate that lines having lower canopy could be better adapted to intermittent stress conditions (Ratnakumar and Vadez 2011) by limiting the effect of stress on reproduction, and thereby the link with HI. Seven QTL were mapped in the region GM1418-S11 (34.3 cM) on LG_AhXVII for the traits HI, SLA and SCMR, while the GM1021-GM1570 region $(21.3 \mathrm{cM})$ on LG_AhXIX contained three QTL for TDW, SCMR and T traits.

Apart from the above, three clusters on LG_AhIV (pPGSSeq19D06-PM418 and TC1D02-TC3E05) and LG_AhVIII (pPGPSeq3A06-IPAHM406) harboring a total of 23 QTL were observed for drought-related traits. The clusters present on LG_AhIV have QTL explaining phenotypic variance of $3.91-33.36 \%$ for traits like SLA, ISC, T, SCMR, TDW etc. The TC1D02-TC3E05 region harboured a QTL for SCMR from RIL-1, which can be taken as a proxy for nitrogen status. It was interesting to find that the same genomic region also harbours QTL for biomass parameters from RIL-2 and RIL-3. Another region, pPGSSeq19D06-PM418 on LG_AhIV, not only harbours a QTL for SLA, which represents processes of leaf thickening, but also has a QTL for LA and transpiration rate (ISC04, in $\mathrm{g}$ water used $\mathrm{cm}^{-2} \mathrm{~h}^{-1}$ ), which represents leaf conductance. Leaf conductance is important for drought adaptation (Kholová et al. 
2010a, 2010b; Zaman-Allah et al. 2011a, b), depending on the stress intensity, as it partly drives plant transpiration, and depends on the degree of leaf expansion (leaf area) and thickening (SLA). These speculations gain support from the fact that a QTL for transpiration (T) from the RIL-1 study was also found in the same region.

Two clusters for yield-related traits with 25 QTL on LG_AhV (GM630-TC6E01) and LG_AhX (TC9F04TC4D09) were also observed with explained phenotypic variance ranging from 1.7 to $13.44 \%$. The genomic region GM630-TC6E01 contained 18 QTL for traits such as PodWt/pl, SeedWt, TDM, HaulmWt and $\mathrm{T}$. It is important to mention here that these QTL have come from different phenotyping experiments. Co-mapping of QTL for yield and component traits with QTL for shoot biomass and transpiration from different experiments underlines the fact that the stress effect in the field experiment of RIL-1 was very mild (200 $\mathrm{mm}$ of rain received during the stress period) and therefore traits related to growth were mostly related to high yield performance. These observations are also re-confirmed by the fact that QTL for pod and seed weight under WW and WS conditions have been comapped in the same region. Although the region between GM2584 and pPGSSeq17F06 on LG_AhV is relatively large $(74 \mathrm{cM})$, it harbours HI QTL from RIL-3 as well as T and shoot biomass QTL from RIL2. These observations have given support to the hypothesis in a separate study (Ratnakumar and Vadez 2011) that genotypes with a smaller canopy can better fare under intermittent drought stress. Such clusters, therefore, can be considered as hotspot genomic regions for genetic dissection to identify tightly linked markers for QTL with high phenotypic variation, as well as for their introgression, if possible, in the same genetic background for improving crop productivity under water-stress conditions.

\section{Conclusions}

In summary, this study reports the first dense consensus map for cultivated groundnut including QTL related to drought-tolerance-related traits. While the present study reports identification of a total of $153 \mathrm{M}$ QTL and 25 E-QTL for drought tolerance, 16 candidate genomic regions harboring 125 QTL related to biomass, yield and drought component traits have been identified for further exploration and utilization for QTL pyramiding and cloning. For the complex traits such as biomass, yield and drought tolerance which are controlled by several genes, many QTL with low to moderate phenotypic variance have been identified that can only be utilized through modern breeding approaches such as MARS or GS. Furthermore, the new genetic maps and consensus map developed will help the groundnut community to align genetic and physical maps in future; in addition they will facilitate the use of the mapped markers for genetic diversity studies, gene/QTL mapping and marker-assisted breeding.

Acknowledgments Thanks are due to Mr. B.J. Moss and Mr. G. Somaraju for conducting lab experiments. Financial support from the National Fund of Indian Council of Agricultural Research (NBFSRA), New Delhi, India; the Tropical Legume I-Objective 1 project of CGIAR Generation Challenge Programme (http://www.generationcp.org), Mexico; the Bill and Melinda Gates Foundation (BMGF), USA; and the Consultative Group on International Agricultural Research (CGIAR) is gratefully acknowledged.

Open Access This article is distributed under the terms of the Creative Commons Attribution Noncommercial License which permits any noncommercial use, distribution, and reproduction in any medium, provided the original author(s) and source are credited.

\section{References}

Ablett GA, Karakousis A, Banbury L, Cakir M, Holton TA, Langridge P, Henry RJ (2003) Application of SSR markers in the construction of Australian barley genetic maps. Aust J Agric Res 54:1187-1195

Bernardo R (2008) Molecular markers and selection for complex traits in plants: learning from the last 20 years. Crop Sci 48:1649-1664

Blum A (2005) Drought resistance, water-use efficiency, and yield potential: are they compatible, dissonant, or mutually exclusive? Aust J Agric Res 56:1159-1168

Collins NC, Tardieu F, Tuberosa R (2008) Quantitative trait loci and crop performance under abiotic stress: where do we stand? Plant Physiol 147:469-486

Cuc LM, Mace ES, Crouch JH, Quang VD, Long TD, Varshney RK (2008) Isolation and characterization of novel microsatellite markers and their application for diversity assessment in cultivated groundnut (A. hypogaea). BMC Plant Biol 8:55

Devi MJ, Sinclair TR, Vadez V (2011) Genotypic variation in peanut for transpiration response to vapor pressure deficit. Crop Sci 50:191-196

FAO (2009) FAO statistical database. http://fastat.fao.org/ 
Frary A, Fulton TM, Zamir D, Tanksley SD (2004) Advanced backcross QTL analysis of a Lycopersicon esculentum $9 \mathrm{~L}$. pennellii cross and identification of possible orthologs in the Solanaceae. Theor Appl Genet 108:485-496

Gupta PK, Varshney RK, Sharma PC, Ramesh B (1999) Molecular markers and their applications in wheat breeding. Plant Breed 118:369-390

Hong Y, Chen X, Liang X, Liu H, Zhou G, Li S, Wen S, Holbrook CC, Guo B (2010) A SSR-based composite genetic linkage map for the cultivated peanut (A. hypogaea L.) genome. BMC Plant Biol 10:17

Hopkins MS, Casa AM, Wang T, Mitchell SE, Dean RE, Kochert GD, Kresovich S (1999) Discovery and characterization of polymorphic simple sequence repeats (SSRs) in peanut. Crop Sci 39:1243-1247

Hubick KT, Farquhar GD, Shorter R (1986) Correlation between water-use efficiency and carbon isotope discrimination in diverse peanut (Arachis) germplasm. Aust J Plant Physiol 13:803-816

Isobe S, Nakaya A, Tabata S (2007) Genotype matrix mapping: searching for quantitative trait loci interactions in genetic variation in complex traits. DNA Res 14:217-225

Jannink J (2007) Identifying quantitative trait locus by genetic background interactions in association studies. Genetics 176:553-561

Karakousis A, Gustafson JP, Chalmers KJ, Barr AR, Langridge P (2003) A consensus map of barley integrating SSR, RFLP, and AFLP markers. Aust J Agric Res 54:1173-1185

Khedikar YP, Gowda MVC, Sarvamangala C, Patgar KV, Upadhyaya HD, Varshney RK (2010) A QTL study on late leaf spot and rust revealed one major QTL for molecular breeding for rust resistance in groundnut (A. hypogaea $\mathrm{L}$.). Theor Appl Genet 121:71-984

Kholová J, Hash CT, Kočová M, Vadez V (2010a) Constitutive water conserving mechanisms are correlated with the terminal drought tolerance of pearl millet (Pennisetum americanum L.). J Exp Bot 61:369-377

Kholová J, Hash CT, Kumar LK, Yadav RS, Kočová M, Vadez V (2010b) Terminal drought tolerant pearl millet [Pennisetum glaucum (L.) R. Br.] have high leaf ABA and limit transpiration at high vapor pressure deficit. J Exp Bot 61:1431-1440

Klimenko I, Razgulayeva N, Gau M, Okumura K, Nakaya A, Tabata S, Kozlov NN, Isobe S (2010) Mapping candidate QTLs related to plant persistency in red clover. Theor Appl Genet 120:1253-1263

Kosambi DD (1944) The estimation of map distance from recombination values. Ann Eugen 12:172-175

Kottapalli KR, Burow MD, Burow G, Burke J, Puppala N (2007) Molecular characterization of the U.S. peanut mini core collection using microsatellite markers. Crop Sci 47:1718-1727

Krishna GK, Zhang J, Burow M, Pittman RN, Delikostadinov SG, Lu Y, Puppala N (2004) Genetic diversity analysis in Valencia peanut (A. hypogaea L.) using microsatellite markers. Cell Mol Biol Lett 9:685-697

Krishnamurthy L, Vadez V, Jyotsna Devi M, Serraj R, Nigam SN, Sheshshayee MS, Chandra S, Aruna R (2007) Variation in transpiration efficiency and its related traits in a groundnut (A. hypogaea L.) mapping population. Field Crops Res 103:189-197
Lander ES, Green P, Abrahamson J, Barlow A, Daly MJ (1987) MAPMAKER: an interactive computer package for constructing primary genetic linkage maps of experimental and natural populations. Genomics 1:174-181

Nageswara Rao RC, Wright GC (1994) Stability of the relationship between specific leaf area and carbon isotope discrimination across environments in peanut. Crop Sci 34:98-103

Nigam SN, Chandra S, Rupa Sridevi K, Manohar B, Reddy AGS, Nageswara Rao RC, Wright GC, Reddy PV, Deshmukh MP, Mathur RK, Basu MS, Vasundhara S, Vindhiya Varman P, Nagda AK (2005) Efficiency of physiological trait-based and empirical selection approaches for drought tolerance in groundnut. Ann Appl Biol 146:433-439

Ratnakumar P, Vadez V (2011) Water use patterns, root characteristics, and yield components under intermittent drought stress in groundnut (A. hypogaea L.) (Unpublished)

Ravi K, Vadez V, Isobe S, Mir RR, Guo Y, Nigam SN, Gowda MVC, Radhakrishnan T, Bertioli DJ, Knapp SJ, Varshney RK (2011) Identification of several small main-effect QTLs and a large number of epistatic QTLs for drought tolerance related traits in groundnut (Arachis hypogaea L.). Theor Appl Genet 122:1119-1132

Rebetzke GJ, Condon AG, Richards RA, Farquhar GD (2002) Selection for reduced carbon isotope discrimination increases aerial biomass and grain yield of rain fed bread wheat. Crop Sci 42:739-745

Ribaut JM, Ragot M (2007) Marker-assisted selection to improve drought adaptation in maize: the backcross approach, perspectives, limitations, and alternatives. J Exp Bot 58:351-360

Ribaut JM, Hoisington DA, Deutsch JA (1996) Identification of quantitative trait loci under drought conditions in tropical maize. 1. Flowering parameters and the anthesis-silking interval. Theor Appl Genet 92:905-914

Ribaut JM, Jiang C, Gonzalez-de-Leon D, Edmeades GO, Hoisington DA (1997) Identification of quantitative trait loci under drought conditions in tropical maize. 2. Yield components and marker assisted selection strategies. Theor Appl Genet 94:887-896

Ribaut JM, de Vicente MC, Delannay X (2010) Molecular breeding in developing countries: challenges and perspectives. Curr Opin Plant Biol 13:213-218

Rucker KS, Kvien CK, Holbrook CC, Wood JE (1995) Identification of peanut genotypes with improved drought avoidance traits. Peanut Sci 22:14-18

Sarvamangala C, Gowda MVC, Varshney RK (2011) Identification of quantitative trait loci for protein content oil content and oil quality for groundnut (A. hypogaea L.). Field Crops Res 122:49-59

Somers DJ, Isaac P, Edwards K (2004) A high-density microsatellite consensus map for bread wheat (Triticum aestivum L.). Theor Appl Genet 109:1105-1114

Stam P (1993) Construction of integrated genetic linkage maps by means of a new computer package JoinMap. Plant J 3:739-744

Varshney RK, Dubey A (2009) Novel genomic tools and modern genetic and breeding approaches for crop improvement. J Plant Biochem Biotechnol 18:127-138 
Varshney RK, Graner A, Sorrells ME (2005) Genic microsatellite markers in plants: features and applications. Trends Biotechnol 23:48-55

Varshney RK, Hoisington DA, Tyagi AK (2006) Advances in cereal genomics and applications in crop breeding. Trends Biotechnol 24:490-499

Varshney RK, Bertioli DJ, Moretzsohn MC, Vadez V, Krishnamurty L, Aruna R, Nigam SN, Ravi K, He G, Knapp SJ, Hoisington DA (2009a) The first SSR based genetic linkage map for cultivated groundnut (A. hypogaea $\mathrm{L}$.). Theor Appl Genet 118:729-739

Varshney RK, Mahendar T, Aruna R, Nigam SN, Neelima K, Vadez V, Hoisington DA (2009b) High level of natural variation in a groundnut (A. hypogaea L.) germplasm collection assayed by selected informative SSR markers. Plant Breed 128:486-494

Wang S, Basten CJ, Zeng ZB (2007) Windows QTL Cartographer 2.5. http://statgen.ncsu.edu/qtlcart/WQTLCart.htm

Xiao J, Li J, Grandillo S, Ahn SN, Yuan L, Tanksley SD, McCouch SR (1998) Identification of trait-improving quantitative trait loci alleles from a wild rice relative, Oryza rufipogon. Genetics 50:899-909

Yang J, Hu CC, Ye XZ, Zhu J (2005) QTLNetwork 2.0. Available at http://ibi.zju.edu. cn/software/qtlnetwork. Institute of Bioinformatics, Zhejiang University, Hangzhou, China

Yonghui Wu, Close TJ, Lonardi S (2008) On the accurate construction of consensus genetic maps. In: Proceedings of LSS computational systems. Bioinformatics conference, 26-29 August 2008, Stanford Edited by: Peter Markstein, pp 285-296
Yoon DB, Kang KH, Kim HJ, Ju HG, Kwon SJ, Suh JP, Jeong OY, Ahn SN (2006) Mapping quantitative trait loci for yield components and morphological traits in an advanced backcross population between Oryza grandiglumis and the O. sativa japonica cultivar Hwaseongbyeo. Theor Appl Genet 112:1052-1062

Young ND, Weeden NF, Kochert G (1996) Genome mapping in legumes (Fam. Fabaceae). In: Paterson AH (eds) Genome mapping in plants. Landes Company, Austin, USA, pp 211-227

Zaman-Allah M, Jenkinson DM, Vadez V (2011a) Chickpea genotypes contrasting for seed yield under terminal drought stress in the field differ for traits related to the control of water use. Funct Plant Biol 38:270-281

Zaman-Allah M, Jenkinson DM, Vadez V (2011b) A conservative pattern of water use, rather than deep or profuse rooting, is critical for the terminal drought tolerance of chickpea. J Exp Bot 62:4239-4252

Zeng ZB (1993) Theoretical basis of separation of multiple linked gene effects in mapping quantitative trait loci. Proc Natl Acad Sci 90:10972-10976

Zeng ZB (1994) Precision mapping of quantitative trait loci. Genetics 136:1457-1468

Zhang J, Zheng HG, Aarti A, Pantuwan G, Nguyen TT, Tripathy JN, Sarial AK, Robin S, Babu RC, Nguyen BD, Sarkarung S, Blum A, Nguyen HT (2001) Locating genomic regions associated with components of drought resistance in rice: comparative mapping within and across species. Theor Appl Genet 103:19-29 